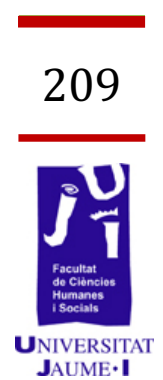

\title{
El cuerpo en movimiento, el método de Verónica Sherborne para la inclusión
}

Marta Prades Monfort marta_mpm2@hotmail.com

María Ángeles Llopis Nebot mallopis@uji.es 
Esta investigación tiene como principal objetivo dar a conocer el método del Movimiento del Desarrollo de Verónica Sherborne en nuestro país. Dicho método es ampliamente conocido y trabajado en diversos países europeos como Gran Bretaña o Polonia, sin embargo no sucede así en España, donde tanto la autora como el método son prácticamente desconocidos.

El método Sherborne consiste en realizar una serie de actividades físicas en pareja o en grupo, mediante las cuales se trabaja la conciencia de uno mismo y de los demás con fines inclusivos y socializadores. Esta metodología se puede utilizar desde la infancia hasta la senectud, para personas con y sin discapacidad, aunque resulta especialmente útil para aquellas con necesidades específicas de apoyo educativo.

En la investigación se ha realizado una revisión bibliográfica sobre Verónica Sherborne, considerando el contexto social donde se desarrolló el método y los antecedentes que influenciaron su obra, así como el trabajo de otros autores a cerca del tema.

Con el fin de estudiar sus beneficios reales, mediante el método de observación participante, se ha analizado el progreso de 25 niños y niñas de educación infantil en un centro escolar de Polonia durante el curso académico 2013/2014. El instrumento para la recogida de datos ha sido el cuestionario de Marta Bogdanowicz, Scale of Behaviour Observation for Children.

Los resultados más relevantes tras analizar la información han sido, por un lado, una notable evolución en el desarrollo cognitivo y emocional de los niños y niñas, si bien a nivel motriz el progreso ha sido algo más modesto. Por otro lado, se ha constatado un aumento de la creatividad y la confianza, tanto a nivel personal como grupal.

Por último, resaltar desde una perspectiva inclusiva, la aportación innovadora que este método podría proporcionar a los programas de educación españoles.

Palabras clave: Sherborne, Movimiento del Desarrollo, Inclusión, Teoría del Movimiento.

\section{Introducción}

La presente investigación pretende dar a conocer en nuestro país el Movimiento del Desarrollo de Verónica Sherborne y su contribución en el desarrollo evolutivo de los niños y niñas, ofreciendo además una constatación práctica del método y su comparación con la teoría, diferenciándose así de otros trabajos realizados a cerca del tema.

Sobre la metodología Sherborne, cabe destacar dos aspectos: En primer lugar, su enfoque inclusivo, que demuestra que es apta para cualquier tipo de alumnado, sobre todo para aquel con NEAE. Y en segundo lugar, por su dimensión holística, diferenciándose de otro tipo de 
metodologías basadas en las habilidades básicas y específicas o en la coordinación óculo-manual. Desde esta concepción, el cuerpo se trabaja como un todo.

\subsection{Los orígenes del Movimiento del Desarrollo de Verónica Sherborne.}

El Movimiento del Desarrollo de Verónica Sherborne, en inglés "Sherborne Developmental Movement" (SDM, en adelante), es un método de enseñanza y de trabajo con movimiento corporal creado en 1960 por Verónica Sherborne (1920-1992), basado en la teoría del análisis del movimiento de Rudolf Laban (1879-1958). Sherborne, aprendió a trabajar esta metodología desde la propia experiencia y la intuición, ya que se consideraba a sí misma una profesional más que una investigadora. En sus propias palabras:

A través de mi experiencia de enseñar y observar del movimiento humano y el aprendizaje a través del ensayo y error, he llegado a la conclusión de que todos los niños tienen dos necesidades básicas; necesitan sentirse en casa con su propio cuerpo y así obtener el dominio, y necesitan ser capaces de establecer relaciones. (Sherborne, 1990).

Sherborne fue una estudiante brillante de educación física y danza, que se interesó por las teorías de Laban, con quien pasó tiempo formándose y aprendiendo, hasta desarrollar su propia teoría y metodología.

La teoría del movimiento de Rudolf Laban (LMA, en adelante), desarrollada en 1948, es uno de los sistemas más utilizados en el análisis del movimiento del cuerpo humano. Este autor describe un sistema complejo capaz de establecer conexión entre las diferentes partes del cuerpo, la dinámica del movimiento corporal, y la interpretación del movimiento (Sandlos, 1999).

Esta teoría se basa en tres componentes principales: la dirección espacial hacia la que el cuerpo se mueve (atrás-adelante, alto-bajo, o de lado), la parte del cuerpo que está en movimiento (de manera sucesiva o simultánea, coordinada en su conjunto o sólo en las extremidades), y el modo en que se mueve el cuerpo (energía, fluidez, espacio y tiempo). La LMA es una metodología utilizada frecuentemente por profesionales cuyo trabajo con el físico es esencial como bailarines, atletas y fisioterapeutas.

Verónica Sherborne basándose en el trabajo de Laban, centró su atención en mejorar las necesidades de los niños y niñas a través de la exploración del propio cuerpo, la expresión corporal ante sentimientos internos y los impulsos al realizar actividades de movimiento. Dicho con sus propias palabras:

De Laban he aprendido una comprensión y conocimiento del cuerpo humano diferente. En mi propio trabajo he aplicado las teorías de Laban a las necesidades de los niños con necesidades especiales y a los niños en edad preescolar. (Sherborne, 2001)

Sherborne explica la teoría del movimiento de Laban mediante un diagrama (figura 1). En éste, el círculo representa la parte central del 
cuerpo y ambas extremidades. La parte inferior hace referencia al conocimiento del cuerpo en gravedad (trabajando con ésta o estableciendo resistencia). En la parte de la izquierda del diagrama, Sherborne enumera las seis direcciones del espacio (arriba-abajo, derechaizquierda, y delante-atrás). En la parte superior la autora apunta los tipos de relaciones que se establecen en el método (por parejas, grupos de 3 ó 4 personas, o el grupo entero, y las relaciones "con", compartidas, o "en contra"). Y por último, a la derecha del esquema están las cuatro dinámicas del movimiento indicadas según en qué rangos pueden variar. El peso, que puede ser entre fuerte y ligero; la fluidez, entre determinada y libre; el espacio, entre directo e indirecto; y el tiempo, entre rápido y prolongado.

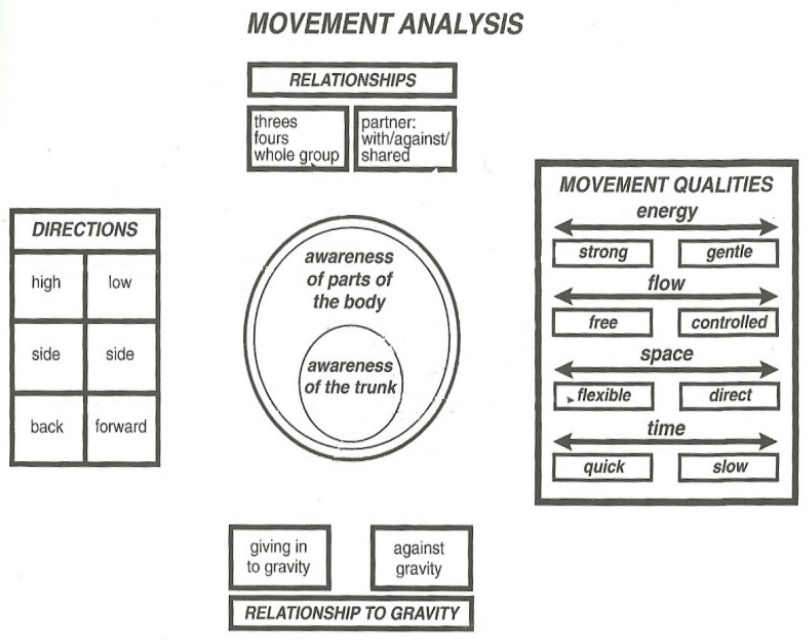

Figura 1. Análisis del movimiento

\subsection{En qué consiste el método Sherborne.}

Verónica Sherborne consideraba que en el desarrollo del ser humano es tan importante el conocimiento del cuerpo como la oportunidad de mantener relaciones sociales, y afirma que el ejercicio es una manera natural de lograr ambas cosas. Por ello, el SDM consiste en realizar una serie de actividades físicas mediante las cuales se trabaja la conciencia de uno mismo y la conciencia de los demás. La conciencia de uno mismo se logra a través de experiencias de movimiento, que ayudan a la persona a ser consciente de lo que sucede en su propio cuerpo a través del tacto y la focalización en sensaciones físicas internas. Estas experiencias ayudan a disminuir la autocrítica y permiten mejorar la autoestima y la confianza. Por otro lado, para desarrollar la conciencia de los demás, el individuo debe aprender a moverse e interactuar con otras personas, construyendo relaciones positivas a través de actividades compartidas.

Para esta metodología, los movimientos en sí no son tan importantes como la manera en que son llevados a cabo, la calidad de las relaciones creadas durante las sesiones y el efecto positivo que todo esto produce en 
el niño o niña (Sherborne, 2001). A través del SDM se pretende crear en el infante sentimientos de valor y respeto, desarrollar autoconfianza y autoestima, y desarrollar sensibilidad y empatía hacia los demás, siendo todo ello fundamental en la educación infantil.

Sherborne crea experiencias de movimiento, y las presenta en un entorno abierto, en el que no se juzga a los participantes y donde se busca el logro y éxito de todos ellos.

Es esencial ayudar a los niños a concentrarse en sus experiencias (...) para que sean conscientes de que está pasando en sus cuerpos. En este camino ellos son capaces de aprender de sus experiencias de movimiento. Yo llamo a esto "escuchar" su cuerpo. (Sherborne, 2001)

EI SDM también fue estudiado y revisado por otros autores. Por ejemplo Hill (2006) observa que el SDM es también una forma de intervención terapéutica, que involucra a los participantes en un aprendizaje interactivo, a través de las experiencias de movimiento compartidas. Marsden y Egerton (2007) aseguran que el SDM es un enfoque inclusivo, donde las experiencias de movimiento son fundamentales para el desarrollo psicomotor y particularmente beneficiosas para aquellas personas con necesidades educativas especiales. Según Bergström y Althoff (2000) la efectividad del método Sherborne se debe a la comunicación e interacción entre los participantes. Laasonen (2005) considera que el elemento clave del SDM es que todo el mundo tiene el derecho a aprender, a sentirse cómodo en su propio cuerpo, a mover y utilizar el cuerpo como uno desee y regular su nivel de actividad. Los objetivos de ese método, según este autor, son aprender en un entorno seguro y protegido, actuar en el propio ritmo y en el del grupo, desenvolverse en diferentes roles, tomar decisiones, y ser tan activo e independiente como sea posible. Para conseguir todo esto, el niño o niña recibe el apoyo constante y la orientación de la maestra.

Hill (2006) por su parte, apunta que el SDM puede realizar una contribución valiosa y significativa para los docentes y estudiantes de educación, ya que las actividades propuestas por el método, mejoran el entendimiento y la confianza en uno mismo creando conexión entre el cuerpo y la conciencia emocional. Este autoconocimiento interno permite al individuo autorregularse y autogestionarse y en consecuencia, esto se reflejará en las relaciones interpersonales, incrementando así la empatía hacia los demás.

En cuanto a los destinatarios, el método Sherborne puede ser beneficioso a cualquier edad, desde la infancia hasta la senectud, para personas con y sin discapacidad ya que la experiencia que se proporciona tanto al participante como al facilitador resulta genuina en cualquier caso. Sin embargo, la práctica de las sesiones resulta particularmente beneficiosa para niños y niñas con necesidades específicas de apoyo educativo (NEAE). Así, el método es empleado fundamentalmente para trabajar con niños y niñas en edad escolar, con personas con 
trastornos mentales y/o múltiples discapacidades, e infantes con problemas de apego.

Para trabajar el método Sherborne, óptimo es realizar sesiones mediante combinaciones mixtas de participantes: con adultos y niños, con niños más mayores y niños de menor edad, o con niños con y sin diversidad funcional. Sin embargo, las sesiones también se pueden realizar con niños y niñas de una misma edad.

\subsection{Cómo se trabaja el método.}

Las sesiones deben ser planificadas cuidadosamente en función de las necesidades que tenga el alumnado. Es recomendable realizar una sesión a la semana y es importante que haya regularidad entre las sesiones, ya que sin una continuidad esta metodología pierde sentido. La duración de las sesiones puede variar desde 15 minutos a una hora, dependiendo de la capacidad y nivel de movimiento de los participantes. La duración de cada actividad dependerá de las respuestas y gustos que manifieste el grupo.

Al inicio de cada sesión se prepara a los participantes, la maestra o el maestro explicará brevemente qué se va a realizar mientras el grupo entero se sienta formando circulo a su alrededor. Esta misma pauta servirá para el cierre de la sesión, reuniendo al grupo otra vez y permitiendo que los participantes se relajen y agradezcan la sesión. Volver a unir al grupo realza la conciencia de haber compartido tiempo y espacio con otras personas y de ser un miembro más del grupo. Además, Bogdanowicz (1992) sugiere que si se trabaja con niños y niñas, empezar y finalizar las sesiones con una canción hace que éstos se sientan más cómodos y partícipes del proceso.

Cada sesión debe contener experiencias que enfoquen los siguientes elementos: conciencia corporal, conciencia espacial, relaciones, creatividad, facilidad para experimentar varias cualidades del movimiento y lenguaje.

Para desarrollar las sesiones no se suelen necesitar materiales, exceptuando mantas o alfombras.

Cuando se trabaje con niños y niñas con diversidad funcional, hay que comprobar con el fisioterapeuta o familiares qué movimientos son seguros y cuales se deben evitar. En conclusión:

Se debe crear un clima de seguridad, un ambiente seguro y proporcionar estímulos positivos que favorezcan la autoexploración. (Sherborne, 2001).

Una sesión modelo del método Sherborne consta de las siguientes partes:

1. Bienvenida. Para abrir la sesión, es recomendable empezar con una canción de bienvenida para integrar a todos los participantes y fomentar la cohesión grupal.

2. Conciencia del cuerpo y del espacio. A continuación, los participantes saludan al grupo con diferentes partes del cuerpo. Por ejemplo: golpean 
el suelo con los pies o las manos, saludan a los compañeros y compañeras," les acarician, etc. El objetivo de esta parte es que el alumnado sea consciente de las diferentes partes del cuerpo y del espacio.

3. Relación "con". El objetivo de este tipo de relación es establecer una primera relación entre las parejas y dar al niño o niña seguridad y confianza para las próximas actividades. Dos roles se distinguen en este tipo de relación: la persona cuidada que tiene un papel pasivo, y la cuidadora que se responsabiliza del bienestar y sensación de seguridad de su pareja.

Este tipo de relaciones exige sensibilidad hacia las necesidades $y$ sentimientos de la persona cuidada, y confianza por parte de la persona cuidada. Además, estas relaciones son beneficiosas porque ayudan a desarrollar una comprensión de la toma de decisiones y la toma de turnos al intercambiar los roles.

Un ejemplo de esta parte de la sesión seria realizar un ligero balanceo lento y suave entre las piernas del cuidador.

4. Relaciones compartidas. Este tipo de relación es similar a la anterior, pero ahora los dos miembros tienen un papel activo, y trabajan cooperativamente para el éxito de la actividad. Estas relaciones requieren confianza, entendimiento, y atención en la pareja. Ayudando a desarrollar una conducta cooperativa.

5. Relación "en contra". En este tipo de relación los participantes prueban su fortaleza contra la resistencia de su pareja. El objetivo es ayudar al niño o niña a focalizar su fuerza, a canalizar la energía, y a desarrollar determinación y concentración, puesto que es importante para los niños y niñas aprender a controlar la fuerza y a saber utilizarla de una forma apropiada.

La relación "en contra", requiere una conciencia constante de la otra persona y sensibilidad para saber cuándo darse por vencido. Si la actividad se realiza con u niño o niña y una persona más mayor, ésta última ajustará su fuerza para que el niño o niña tenga siempre sensación de éxito.

Este tipo de actividades se deben tomar como un juego y afrontarlas con humor, sin agresión.

6. Relajación. Relajación. En este apartado, los participantes realizan un pequeño masaje a su pareja o la balancean suavemente con el objetivo de relajarse después de la actividad energética. 
7. Relación "juntos". En esta parte de la sesión, la actividad se desarrolla con" la participación de todo el grupo. Fomentando así el trabajo cooperativo 216 y la conciencia de pertenencia al grupo.

8. Relajación y despedida. Para cerrar la sesión, los participantes cantan una canción de despedida, mientras se relajan tumbados en el suelo con los ojos cerrados.

Cabe destacar que el transcurso de una actividad a otra se realiza mediante desplazamientos por el suelo, brindando así a los participantes la oportunidad de moverse por un medio poco usual en la escuela.

También es necesario recordar que según los aspectos a trabajar, se llevan a cabo unas actividades $\mathrm{u}$ otras, considerando las necesidades y preferencias del alumnado, e introduciendo gradualmente actividades nuevas para evitar la rutina y el aburrimiento.

\subsection{Extensión del método por el mundo.}

Durante años, Sherborne presentó su trabajo ante docentes y terapeutas de diversos países como: Noruega, Suecia, Canadá, Australia, Eslovaquia, Republica Checa, Polonia, y más tarde en Finlandia, Estonia y Bélgica. Varios de estos profesionales, convencidos de las ventajas del método para el desarrollo físico y social de los niños y niñas, decidieron implantarlo en sus escuelas y darlo a conocer a otros profesionales de sus países.

Desde la muerte de Verónica Sherborne en 1990, la formación y el interés profesional sobre el método no ha dejado de crecer y expandirse por otros países europeos y asiáticos como Japón. En reconocimiento de su trabajo y su memoria, se han fundado las Asociaciones Sherborne en el Reino Unido, Bélgica, Suiza, y en otros muchos países como Australia, Brasil, Canadá, Finlandia, Alemania, Italia, Japón, Holanda, Noruega y Polonia.

EI SDM es un método abierto, que su propia autora desarrolló bajo la propia experiencia práctica y por lo tanto, cada docente debe adaptarlo a sus propias necesidades. En palabras de la autora:

Yo realizo mi trabajo y tú tomas mis ideas, utilizándolas de la forma que creas más conveniente. Tú creas tu propio camino de trabajo. (Sherborne, 1990)

En cuanto a la situación del método Sherborne en España, si bien existe conocimiento sobre el trabajo de Rudolf Laban y se pueden encontrar varias de su publicaciones en lengua castellana como El dominio del movimiento (Laban, 1987), Danza educativa moderna (Laban, 1978), La Cinetografía Laban (Naranjo, 2004) entre otros, la bibliografía sobre el método Sherborne es inexistente, resultando prácticamente imposible hallar experiencias o casos de aulas españolas donde se haya trabajado la metodología Sherborne. 
III. Objetivos

El objetivo principal de esta investigación es describir el método de Movimiento del Desarrollo de Verónica Sherborne y darlo a conocer en España.

También se pretende comprobar si la aplicación práctica sistemática de esta metodología en el aula supone beneficios evolutivos y/o emocionales en niños y niñas.

\section{Material y método}

La muestra de esta investigación está compuesta por 25 alumnos, 9 niñas y 16 niños de 5-6 años de edad, pertenecientes al colegio público "Przedszkole nr 2 im. Jana Brzechwy" en Gdansk (Polonia). Las sesiones SDM se han realizado con el acompañamiento de 9 adultos más la profesora experta en el método Sherborne.

La metodología utilizada para la intervención ha sido la observación participante de las sesiones, realizadas una vez por semana durante el curso escolar 2013/2014. Se han realizado 25 sesiones de observación en total.

El instrumento empleado para la recogida de información ha sido el cuestionario de observación "Scale of Behaviour Observation for Children" de Marta Bogdanowicz, que consta de 20 ítems en los que se valora del 1 al 5 la consecución de los objetivos planteados. La plantilla de observación ha sido pasada a los niños y niñas en dos ocasiones, al principio y al final del curso académico para comprobar su progreso físico y social.

\section{Resultados}

Tras analizar la información recogida en las plantillas de observación, se han podido extraer los resultados que se detallan a continuación.

En autonomía e iniciativa personal, los niños y niñas han mejorado la coordinación, la flexibilidad, su eficiencia motora, las habilidades de control y manipulación, la expresión corporal, la creatividad y tienen menos dependencia de los adultos durante las actividades motoras.

En comunicación lingüística, el alumnado ha mejorado la comunicación debido al continuo dialogo existente entre los participantes, a través de varios canales como: el intercambio verbal, el lenguaje corporal, el contacto visual, las expresiones faciales, o los gestos. Además, los niños y niñas han aprendido a incorporar cualidades del movimiento, acciones y vocabulario sobre nociones espaciales y partes del cuerpo en su léxico, no solo en su lengua materna (polaco), sino también en lengua inglesa. 
En competencia social y ciudadana, el alumnado ha tenido la oportunidad de desarrollar la personalidad (sentimientos hacia uno mismo y hacia los demás), las habilidades interpersonales y el trabajo cooperativo. Se ha constatado cómo el alumnado aumentaba la cantidad de interacción con su pareja, disminuía la inseguridad hacia el contacto físico, se relajaba más fácilmente, expresaba mejor sus emociones y estaba más motivado para experimentar y realizar nuevas actividades.

El SDM también ofrece mejorías en aspectos relacionados con la competencia matemática, ya que muchas actividades focalizan la atención sobre la postura del cuerpo, los tamaños y las formas. Los infantes han mejorado la noción de dirección, conceptos espaciales, secuencias del movimiento y conocimiento sobre números, formas y tamaños de los grupos.

Respecto al conocimiento y la interacción con el mundo físico, los participantes han mejorado aspectos como control de la fuerza, conciencia corporal, velocidad y conocimiento de las partes corporales.

Como se puede observar en la Figura 2, donde se comparan varios ítems al inicio y fin del curso académico, la toma de turnos, el contacto visual, la atención, el trabajo cooperativo, el reconocimiento de las diferentes partes del cuerpo, y la coordinación han aumentado al final de la intervención. Mientras que tanto la imitación como la desvinculación han disminuido, indicando así que cada vez los niños y niñas son más autónomos y activos en las sesiones.

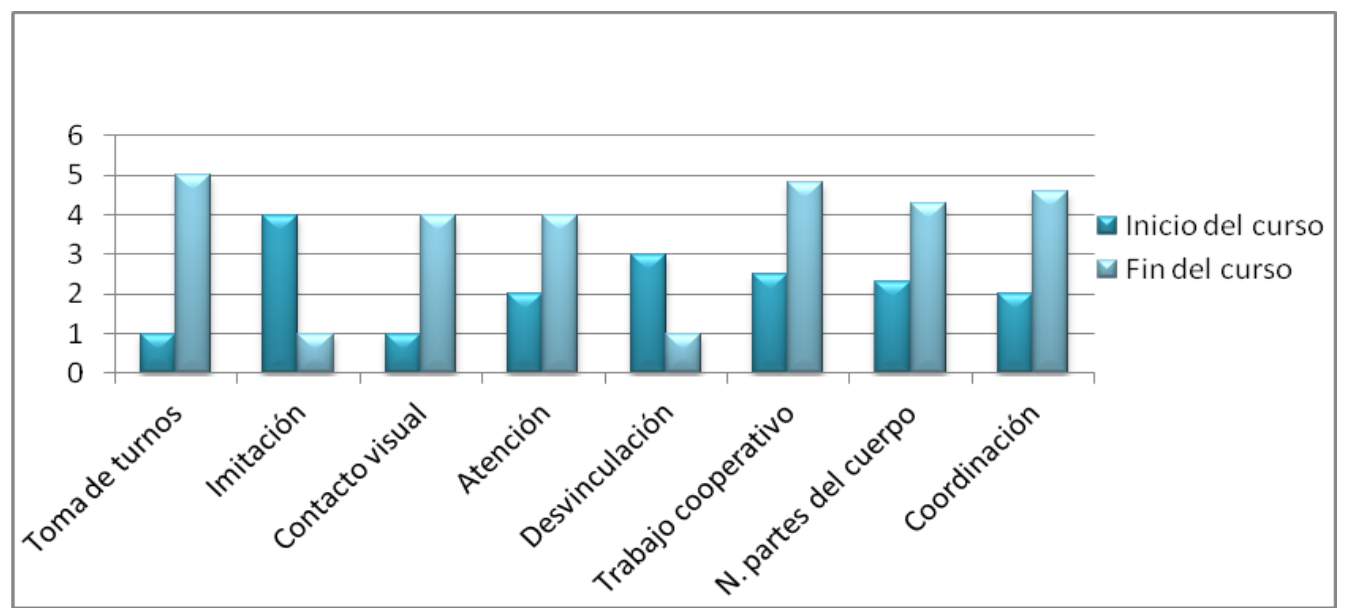

Figura 2. Progreso del alumnado durante el curso. 
En la figura 3 se muestra cómo ha evolucionado el desarrollo cognitivo, emocional y motor de los niños y niñas durante las sesiones SDM. El aspecto emocional es el que más ha notado la subida, ya que casi duplica su valor al finalizar el curso. El desarrollo motor y cognitivo del alumnado también han mejorado, aunque no en unos valores tan altos

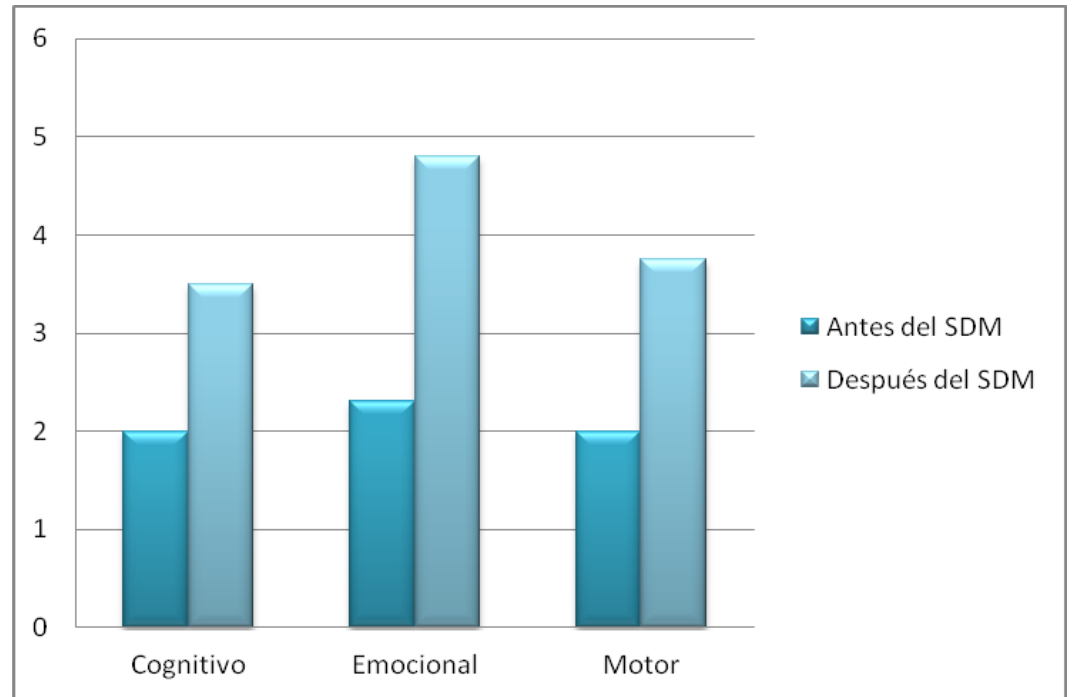

Figura 3. Gráfico sobre el desarrollo global del alumnado

Se ha analizado también qué tipo de relaciones ofrecen mayor dificultad a los participantes, siendo las relaciones "con" y las relaciones compartidas las más sencillas mientras que las relaciones "en contra" resultan más complicadas al necesitar mayor autocontrol y canalización de energía.

Por último, también se investigó el nivel de satisfacción de las familias con el método Sherborne. Los resultados se muestran en la figura 4. Se puede observar que un $85 \%$ de las familias opina que el SDM resulta muy útil para el desarrollo de los niños y niñas, un $13 \%$ piensa que es útil y un $2 \%$ es neutral en cuanto a su utilidad.

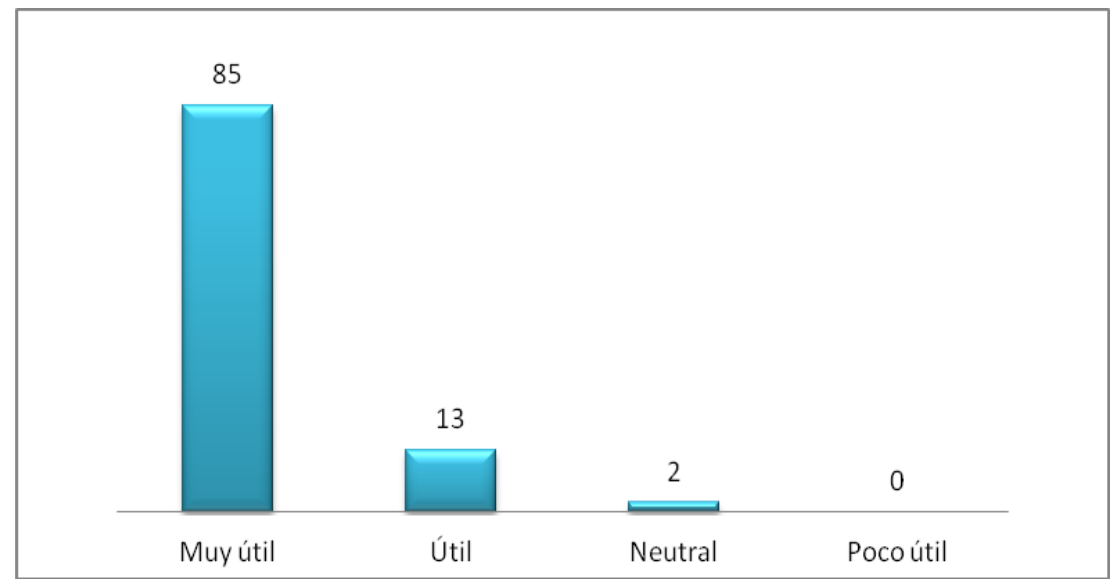

Figura 4. Nivel de satisfacción de las familias sobre el SDM 
En conclusión, el SDM ha ofrecido a los participantes de las sesiones la oportunidad de crecer física, social, emocional e intelectualmente, adquiriendo además mayor creatividad y confianza tanto en uno mismo como en los demás y resultando altamente útil para las familias de los y las participantes.

\section{Discusión y conclusiones}

El método Sherborne es una pedagogía holística, integradora y creativa que tiene mucho que ofrecer a los programas de educación. Entre las ventajas que presenta, cabe destacar su adaptabilidad a las necesidades de cada grupo, pudiendo complementar otras actividades escolares tanto de expresión física como emocional y resultando una herramienta eficaz para la inclusión.

En el desarrollo físico, los usuarios experimentan sentirse cómodos en sus propios cuerpos, aprendiendo control sobre éste generalizando dicho control a diferentes situaciones. En el desarrollo de la personalidad, los niños y niñas adquieren un fuerte sentido de sí mismos, aumenta la seguridad en sus propias habilidades, se favorece la iniciativa e inventiva así como se aumenta la sensibilidad hacia los demás. Mediante la focalización de la atención en la actividad los participantes experimentan sentido de logro y de realización.

Siguiendo a Bleszynski (2004), el SDM es uno de los métodos más comunes como terapia del desarrollo en Europa. Lo más positivo del método es que resulta altamente abierto y moldeable, docentes y terapeutas lo pueden adaptar y ajustar según las necesidades del grupo con el cual se va a trabajar.

El Movimiento del Desarrollo de Sherborne ha sido utilizado durante años como una parte independiente o integrada de las clases en colegios y escuelas infantiles. Filer (2010) defiende el uso terapéutico del método para trabajar dificultades emocionales en la infancia ya que consigue crear un ambiente natural en el cual expresar libremente emociones y percepciones. Ahtiainen (2010), por su parte, hace hincapié en la utilidad del método para desarrollar habilidades que abocan a la maduración física y personal.

Destacar así mismo, el rol de las familias en la práctica del método Sherborne. EI SDM permite incluir a las familias en la práctica de actividades cooperativas, favoreciendo el vínculo paterno-filial a través de la comunicación y la expresión corporal.

Algunas controversias que surgieron ante la novedad del método cuestionaban el posible efecto negativo que podían generar los ejercicios sobre relaciones "en contra", como posibles generadores de agresividad y los ejercicios sobre relaciones "con", a los cuales se les atribuía exceso de contacto físico. Sin embargo, la evidencia posterior ha confirmado sobradamente los beneficios del contacto físico en el desarrollo madurativo, generador de autoconfianza y seguridad, mientras que en las 
relaciones "en contra", es precisamente el aprendizaje del control de la fuerza y la canalización adecuada de la agresividad su mayor beneficio.

Algunas propuestas futuras para la implantación del método en España consistirán, en primer lugar, dar a conocer a través de seminarios, charlas, o congresos de educación el método de Verónica Sherborne. En segundo lugar, proporcionar formación adecuada al profesorado interesado para que éste pudiera comenzar a desarrollar el SDM en las aulas. Debería analizarse de forma paralela las consecuencias de la implantación del método, especialmente las referidas a la satisfacción de la comunidad educativa y de las familias.

La mayor dificultad de implantar el método en España recae sobre la formación del profesorado, ya que no existen expertos en esta metodología en el territorio español, por lo cual, las personas interesadas deberían desplazarse a países donde impartan los cursos o bien se deberían desplazar dichos expertos a nuestro país, con el consecuente coste económico de ambas posibilidades.

En definitiva esta metodología ha resultado útil y eficaz en diversos contextos geográficos, motivo por el cual es esperable que también resulte de interés en territorio español, sin olvidar que el verdadero valor y calidad del SDM solo puede ser apreciado completamente involucrándose en su práctica y formando parte activa en su implantación.

\section{Bibliografía}

Ahtiainen, A. (2010): "Work related challenges and Sherborne developmental movement -educational evening for Vehmaa day care personnel ", Publicaciones de Satakunta University of Applied Sciences, Finland.

BERgStRÖM, A. Y B. ALTHOFF (2000): Sherbourne ett projekt i sinnesstimulering och kommunikation. Recuperado de http://www.vkl.se/filarkiv/vkl/pdf/uou/fou_rapporter/sherborne.pdf BLESZYNSKI, J. (2004): "Application of the Developmental Movement Method in the therapy of a child with Serious Developmental deficiencies", Publicaciones de Nicolaus Copernicus University, Torún.

Bogdanowicz, M. (1992): Metoda Weroniki Sherborne $w$ terapii $i$ wspomaganiu rozwoju dziecka, Wydawnictwa Szkolne i Pedagogiczne, Warszawa.

Bogdanowicz, M. (1993): Materiały dla uczestników kursów Metody Ruchu Rozwijajqcego Weroniki Sherborne, PTD, Gdańsk.

Bogdanowicz, M. (2003): Skale Obserwacji Zachowania (SOZ) Dzieci i Rodziców uczestniczqcych w zajęciach Ruchu Rozwijajqcego Weroniki Sherborne, Wydawnictwo Harmonia, Gdańsk.

Bogdanowicz, M. y otros (1992): Metoda Weroniki Sherborne w terapii $i$ wspomaganiu rozwoju dziecka, WsiP, Warszawa.

FILER, J. (2010): «Developmental Movement Play - Moving into Motion to Transform Lives and Well-being: Using Ourselves to Communicate through Movement», Publicado en Children's workforce development council, UK. 
HILL, C. (2006): Communicating through Movement, Sunfield Publications,

Great Britian.

LAASONEN, K. (2005): "Liikkumisen iloa Sherborne menetelmällä in Rintala», en Ahonen P. (2005): Liiku ja Opi, Liikunnasta apua oppimisvaikeuksiin (pp. 125-154), Otavan Kirjapaino, Keuruu.

LABAN, R (1950): Mastery of Movement, Northcote House Publishers, London.

- (1988): Modern Educational Dance, Northcote House Publishers, London. - (1975): Principles of Dance and Movement Notation, MacDonald, London. - (1978): Danza educativa moderna. Paidós, Barcelona.

- (1987): El dominio del movimiento. Fundamentos, Madrid.

MARSDen, E. y J. EgerTon (2007): Moving wit research: evidence-based practice in Sherborne Developmental Movement, Sunfield Publications, Stourbridge, UK.

MARSDERN, E. (2003): International Sherborne Co-operation (ISCO). Publicado en In touch, Sherborne Association UK.

NARANJo, P. (2004): La cinetografía Laban. Artes, la revista 7.

SANDLOS, L. (1999): Laban Movement Analysis: Unlocking the Mysteries of Movement. Recuperado de http://www.xoe.com/LisaSandlos/labanmovement-analysis

SHERBORNE, V. (1990): Movement and the integration of exceptional children. In The Educational Forum (Vol. 54, No. 1, pp. 105-116). Taylor \& Francis Group.

- (2001): Developmental movement for children, Worth Publishing, London. 